\title{
FLARES AND MHD JETS IN PROTOSTAR
}

\author{
MITSURU HAYASHI \\ Japan Atomic Energy Research Institute(JAERI) \\ 801-1 Mukouyama,Naka-machi,Naka-gun, \\ Ibaraki,311-01,Japan \\ KAZUNARI SHIBATA \\ National Astronomical Observatory \\ Mitaka, Tokyo 181, Japan \\ AND \\ RYOJI MATSUMOTO \\ Department of Physics, Faculty of Science, Chiba University \\ Inage-Ku, Chiba 263, Japan
}

\section{Introduction}

By using ASCA, Koyama et al. $(1994,1996)$ carried out a systematic survey of hard X-ray sources in molecular clouds and revealed that protostars are strong hard X-ray emitting sources. Some of them show flare-like activities. Protostellar flares differ from solar flares in their total energy $\left(10^{35-36} \mathrm{erg}\right)$, size( several times the radius of protostar), and higher temperature( $8 \mathrm{keV}$ ). Protostellar flares are also observed in lower energy band by ROSAT in YLW15 (Grosso et al. 1997). By extending the model of solar flares associated with footpoint shearing motion, we proposed a model of protostellar flares in which the magnetic field connecting the protostar and the disk disrupt by twist injection from the rotating disk(Hayashi et al. 1996).

\section{Numerical Models \& Results}

We solve the resistive MHD equations in clindrical coordinate by applying a modified Lax-Wendroff scheme with artificial viscosity. The effects of radiative cooling and rotation of the central star are neglected. Magnetic field lines connecting the central star and the disk are twisted by the rotation of the disk. As the magnetic twist accumulates, the magnetic loops ex- 
pand quasi-statically in the early stage but later, they expand dynamically. Magnetic reconnection takes place in the current sheet formed inside the expanding loops. Outgoing magnetic island and post flare loops are formed after the reconnection (See Fig 1 in Hayashi et al. 1996).

We carried out longer time-scale simulations to investigate the evolution of the post flare loops created by reconnection event. We found that magnetic reconnection and plasmoid ejection occur intermittently.

\section{Discussion}

We found that the dipole magnetic fields of the protostar can become partially open by imposed twists within one rotation of the disk. We also showed that hot plasmoids are ejected in bipolar directions with velocity 2- 5 times the Keplarian rotation speed $\left(\mathrm{v}_{K_{0}}\right)$ around the inner edge of the disk. Plasma heating occurs both by Joule heating in the current sheet and by shock waves created by magnetic reconnection. The plasma temperature corresponding to a reconnection flow speed $(\sim$ Alfvén speed) of 2-5 $\mathrm{v}_{K 0}$, where $\mathrm{v}_{K 0}$ is the Keplarian velocity at unit radius $\mathrm{r}=\mathrm{r}_{0}=1$, can be $10^{7}$ $10^{8} \mathrm{~K}$, consistent with the observed spectrum which extends up to $10 \mathrm{keV}$. Since magnetic reconnection accelerates electrons, we expect synchrotron radiation from ejected plasmoids. Recently, Hughes (1997) reported that in the young star forming region Cepheus A, nonthermal sources are separating with a relative transverse velocity $(\sim 370 \mathrm{~km} / \mathrm{s})$. In T-Tauri region, Ray et al. (1997) found two lobes which emit radio emission exhibiting strong circular polarization of opposite helicity. These observations are consistent with our reconnection model of hot plasmoid ejections.

The size of the flaring loop is several times the stellar radius. In addition, recently ASCA detected X-ray flaring variability which repeats three times recursively (Tsuboi et al. 1997). This can be the first observatuon which supports intermittent flaring variability in star forming region. Such recurrent activities can be explained by intermittent magnetic reconnection in the disk-star connecting magnetic loop.

\section{References}

Grosso, N., Montmerle, T., Feigelson, E.D., André, P., Cassanova, S., \& Gregorio-Hetem, J. 1997, Nature, 385, 56

Hayashi, M.R., Shibata, K. \& Matsumoto, R. 1996, ApJ, 468, L37

Hughes, V. A., 1997, ApJ, 481, 857

Koyama, K., Hamaguchi, K., Ueno, S., Kobayashi, N., \& Feigelson, E. D. 1996, PASJ, 48, L87

Koyama, K. et al. 1994, PASJ, 46, L125

Ray, T. P., Muxlow, T. W. B., Axon, D. J., Brown, A., Corcoran, D., Dyson, J., \& Mundt, R. 1997, Nature, 385, 415

Tsuboi, Y. et al. 1997 IAU Symposium No.188, "The Hot Universe" 